\title{
Comparison of a Direct and Indirect Method for Measuring Flavins- Assessing Flavin Status in Patients Receiving Total Parenteral Nutrition
}

\author{
J.S. Hamilton ${ }^{1}$, J.V. Woodside ${ }^{2}$, W.A. Mcllveen ${ }^{1}$, M.C. McKinley ${ }^{2}$ and I.S. Young ${ }^{*, 1,2}$ \\ ${ }^{I}$ Department of Clinical Biochemistry, Royal Victoria Hospital, Grosvenor Road, Belfast BT12 6BA, UK \\ ${ }^{2}$ Nutrition and Metabolism Group, Centre for Clinical and Population Science, Queen's University Belfast, Grosvenor \\ Road, Belfast BT12 6BJ, UK
}

\begin{abstract}
Background: Riboflavin, flavin mononucleotide (FMN) and flavin adenine dinucleotide (FAD) are cofactors in oxidation-reduction reactions. The aim of this study was to compare a direct high performance liquid chromatography (HPLC) method with an indirect enzymatic method to assess flavin status and establish reference intervals. The HPLC method was used to assess prevalence of flavin deficiency in total parenteral nutrition (TPN) patients.

Method: Blood was obtained from 90 healthy volunteers and 51 TPN patients. Results for each method were compared using Spearmans Rank correlation. Results from reference groups and TPN patients were compared using Mann-Whitney U tests.

Results: Erythrocyte glutathione reductase activity coefficients (EGRAC) correlated with flavin levels measured by HPLC $(\mathrm{p}<0.001)$. HPLC was chosen for TPN samples analyses due to superior sensitivity. There was no significant difference between FAD and FMN levels in controls and TPN patients, although TPN patients had significantly higher levels of riboflavin than controls $(\mathrm{p}<0.001)$.

Conclusion: HPLC is superior to the EGRAC assay for assessing flavin status. Hyporiboflavinosis is not prevalent in TPN patients.
\end{abstract}

\section{INTRODUCTION}

Clinical signs of severe flavin deficiency include angular stomatitis, cheilosis, glossitis, and magenta tongue [1]. Riboflavin is an essential component of FMN and FAD, which are involved in oxidation-reduction reactions that occur during energy production $[1,2]$, biosynthesis, detoxification, and electron scavenging pathways $[3,4]$. Riboflavin is involved in iron transport [5-7], activation of other vitamins $[1,5,6]$, and homocysteine metabolism $[1,7,8]$. Riboflavin deficiency causes widespread disturbance in metabolic pathways, interfering with normal cellular function $[3,4]$, thus hindering recovery of patients receiving TPN.

There is no storage pool of riboflavin and deficiency can result from inadequate intake, decreased assimilation due to malabsorption, defective utilisation, drugs, increased destruction, enhanced excretion in catabolic patients, and increased requirements due to physiological and pathological stress such as pregnancy $[1,3]$.

In riboflavin deficiency, characteristic changes occur in the cellular distribution of flavin fractions, and activities of flavin-dependent enzymes. FAD is conserved at the expense of FMN, suggesting a specific hierarchical response to riboflavin deficiency whereby FAD-dependent reactions such as the electron transport chain in ATP synthesis are preserved at the expense of less important pathways [1,9].

*Address correspondence to this author at the Nutrition and Metabolism Group, Centre for Clinical and Population, Queen's University Belfast, Institute of Clinical Science, Grosvenor Road, Belfast BT12 6BJ, UK; E-mail: i.young@qub.ac.uk
It has been suggested that patients on long-term TPN will inevitably develop deficiencies in blood vitamins if not properly supplemented and monitored [10]. However, few studies have been performed to establish the adequacy of supplementation, and most of those involve only neonates [11].

Currently there is debate over the best way to assess riboflavin status. Assessment has been made on the basis of clinical signs, urinary excretion, plasma and erythrocyte content, and erythrocyte glutathione reductase activity (EGRAC) [12]. Clinical signs are difficult to evaluate as severity can vary widely between individuals [13], so the question remains as to which method is most suitable for routine flavin analysis [3,14-16]. Direct HPLC analysis is more sensitive and specific than other methods. However, the most common method used is an indirect functional assay, EGRAC, which is dependent on FAD levels [17]. The assay requires a small non-fasting sample and the enzyme is stable in washed red cells. However, while EGRAC responds quickly to acute riboflavin deficiency, it is unknown if it changes during progressive depletion [9]. There is discord over what constitutes the deficiency threshold and appropriate cut-off point for abnormal EGRAC [1]. Sensitivity and specificity of EGRAC are questionable in that other factors may affect glutathione reductase activity, such as inherited glucose-6-phosphate deficiency and high levels of free fatty acids $[3,4,12,15,18]$.

The aims of this project were to compare a direct HPLC method with an indirect enzymatic method in order to assess flavin levels, establish reference intervals for flavins for each 
method using samples obtained from normal healthy individuals, and assess the adequacy of flavin supplementation in TPN patients.

\section{MATERIALS AND METHODOLOGY}

Healthy individuals ( 52 male, 38 female) aged between 20 and 65 years were recruited from hospital and university staff and students, as well as from outpatient clinics. Exclusion criterion was the use of vitamin supplements containing riboflavin. All participants provided informed written consent, and ethics approval was obtained from Queen's University Belfast Research Ethics Committee. The number of normal subjects recruited was limited by the timeframe of the study and was therefore slightly less than the IFCC recommendations of 120 subjects.

51 patients ( 29 males, 22 females) ranging in age from 18 - 80 years were recruited from the weekly nutrition ward round. The patients were receiving TPN for a variety of causes including short bowel syndrome, post-operative recovery, and intestinal dysfunction against a background of critical illness. The range of causes was too diverse to allow subjects to be further categorised for sub-group analysis.

Fasting venous blood samples were collected from both reference and patient groups in tubes containing EDTA, which has been shown to inhibit the activity of enzymes that hydrolyse FAD and FMN [19], and prepared for analysis under dim light immediately after venepuncture. Plasma was separated and stored at $-80{ }^{\circ} \mathrm{C}$ for analysis by the HPLC method. For the indirect EGRAC assay, red cells were washed in saline and frozen at $-80{ }^{\circ} \mathrm{C}$ prior to analysis. All chemicals were obtained from Sigma Chemical Co. (St Louis, MO, USA) unless otherwise stated.

The EGRAC method is based on the measurement of erythrocyte glutathione reductase activity as described by Sauberlich et al. [17]. Erythrocyte glutathione reductase (EGR) is a flavin enzyme with FAD as a prosthetic group.

$\mathrm{GSSG}+\mathrm{NADPH}+\mathrm{H}^{+} \stackrel{\mathrm{FAD}, \mathrm{EGR}}{\longrightarrow} 2 \mathrm{GSH}+\mathrm{NADP}^{+}$

Its activity is altered in vivo by dietary riboflavin and in vitro by FAD. The degree of in vitro glutathione reductase activity depends on the FAD saturation of the apoprotein, which in turn depends on riboflavin availability. The principle of the assay is that the decrease in absorbance of the reaction mixture at $340 \mathrm{~nm}$ due to the decrease in NADPH concentration is a measure of the conversion of oxidised glutathione (GSSG) to reduced glutathione (GSH), and thus glutathione reductase activity. EGRAC values were calculated using the following equation:

EGRAC $=\frac{\text { Absorbance of solution with added FAD }}{\text { Absorbance of solution without added FAD }}$

The HPLC assay, based on the method of Capo-chichi et al. [4], determines simultaneously riboflavin, FMN, and FAD in plasma using lumiflavin (riboflavin analogue) as an internal standard. All solvents used in this procedure were of HPLC grade (Analytical Supplies Ltd., Dromore, Co Down, $\mathrm{UK})$.

Briefly, mobile phase consisting of $85 \%$ Solution A (15 $\mathrm{mM}$ magnesium acetate and $10 \mathrm{mM}$ potassium phosphate, $\mathrm{pH} 3.4$ ) and $15 \%$ acetonitrile was prepared. A mixture of standards were prepared and analysed with each batch of samples to distinguish the retention times of the flavins. Frozen plasma, along with in-house quality control plasma samples were thawed prior to the addition of lumiflavin and magnesium acetate. Samples were incubated in a water bath to ensure total release of the flavins from their apoenzymes. The samples were deproteinised and the flavins extracted using C18 3cc Sep-Pack cartridges (Waters Corporation, Ireland). The Sep-Pak desalted the supernate and helped avoid interference in the fluorescence intensity of the flavins due to quenching by anions, particularly chloride anions from the deproteinisation step [4]. The samples were then stored at $4{ }^{\circ} \mathrm{C}$ prior to injection onto a C18 RP-HPLC column (C18, $5 \mu \mathrm{M}, 250 \times 4.6$ mm, Hypersil, Alltech, UK).

The flavins were detected using a scanning fluorimeter (Waters Corporation, Ireland) with wavelength settings of $445 \mathrm{~nm}$ excitation and $530 \mathrm{~nm}$ emission, with a flow rate of $1 \mathrm{~mL} / \mathrm{minute}$. Elution of the flavins (See Fig. 1) followed the order of polarity (FAD $>$ FMN $>$ riboflavin> lumiflavin). The fluorimetric signals (peak heights) of the flavins were directly proportional to their concentrations.

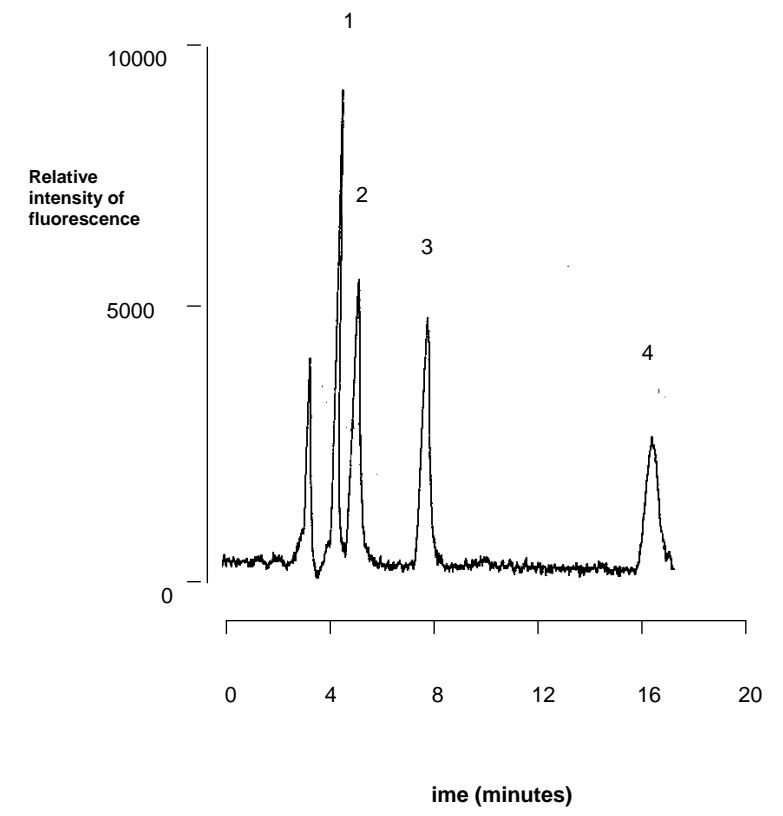

Fig. (1). Chromatogram of the flavins in plasma spiked with $24 \mathrm{nM}$ FAD, $22 \mathrm{nM}$ FMN, $26.5 \mathrm{nM}$ riboflavin, and $39 \mathrm{nM}$ lumiflavin.

Peak 1 with a retention time of approximately 4.23 minutes $=$ FAD, peak 2 at 5.13 minutes $=$ FMN, peak 3 at 7.77 minutes $=$ riboflavin, peak 4 at 16.57 minutes $=$ lumiflavin.

The sensitivity, intra- and inter-assay variation, limit of detection, and \% recovery were calculated for the EGRAC and HPLC methods. SPSS software (version 17, Seattle, USA) was used for all analyses. As the data were not normally distributed, the methods were compared using Spearman's Rank correlation. The reference intervals for both methods were defined as the 2.5 and 97.5 percentiles [20, 21]. The results from the TPN patients were compared with these reference intervals using the Mann-Whitney $U$ test in order to determine whether riboflavin deficiency is a significant problem in this population. $\mathrm{P}$ values $<0.05$ were considered to be significant. 
Table 1. Summary of the Results from Method Evaluation Studies

\begin{tabular}{|l|l|l|}
\hline & HPLC $(\mathbf{n M})$ & EGRAC assay \\
\hline Analytes detected & FAD, FMN, Riboflavin & FAD \\
\hline \hline Limit of Detection (nM) & $\begin{array}{l}\text { FAD }=1.0 \\
\text { FMN }=0.5 \\
\text { Riboflavin }=0.5\end{array}$ & FAD $=7.5$ \\
\hline Linearity & Linear for all flavins & Non-linear \\
\hline Intra-assay CV (\%) & FAD $=2.0$ & FAD $=7.7$ \\
\hline Inter-assay CV (\%) & FMN $=1.3$ & \\
\hline Riboflavin $=4.7$ & FAD $=9.0$ \\
\hline Reference interval & FAD $=9.6$ & \\
& FMN $=9.5$ & \\
& Riboflavin $=9.6$ & EGRAC $=0.93-1.59$ \\
& FAD $=26.2-84.1$ & \\
\hline
\end{tabular}

\section{RESULTS}

\section{Assessment of the Methods}

The intra- and inter-assay variation and limit of detection (LOD) were calculated for both methods, and \% recovery and linearity were calculated for the HPLC method. The LOD was calculated by multiplying the standard deviation of the blank $(n=10)$ by 3.3 and dividing it by the slope of the standard curve. The results for each of the methods are shown in Table $\mathbf{1}$.

\section{HPLC}

The LODs compare well with other HPLC methods which quote FAD at approximately $4.0 \mathrm{nM}$, FMN at approximately $3.0-3.5 \mathrm{nM}$, and riboflavin between $2.9-9.0 \mathrm{nM}$ $[22,23]$. The peak heights of the flavins were directly proportional to their concentrations up to $240 \mathrm{nM}$ FAD, $220 \mathrm{nM}$ FMN, $265 \mathrm{nM}$ riboflavin, and $390 \mathrm{nM}$ lumiflavin, and were thus used to quantify flavin levels. The intra- and inter-assay coefficients of variation (CV) were found to be within acceptable limits $(<10 \%$, intra-assay $n=20$, inter-assay $n=12$ over a 12 week period). Recovery of the samples after the extraction process was between 86 and $94 \%$ for all flavins $(\mathrm{n}=5)$.

\section{EGRAC}

The standard curve for the EGRAC assay was non-linear, suggesting that the assay has greater sensitivity at concentrations of FAD less than approximately $50 \mathrm{nM}$, whereas at higher levels, a large change in concentration would not produce a corresponding change in activity coefficient. Due to the non-linearity of the standard curve, in order to establish the sensitivity of the EGRAC assay, UV-irradiated flavindeficient red cells were spiked with decreasing levels of known FAD concentrations, until the CV became unacceptable. The CV of the assay was within acceptable limits $(<10$
$\%$ ), at concentrations of FAD greater than $7.5 \mathrm{nM}$. The intraassay CV was $7.7 \%(n=20)$ and the inter-assay $\mathrm{CV}$ was $9 \%$ at a FAD concentration of $40 \mathrm{nM}(\mathrm{n}=12$ over a 12 week period).

\section{Establishing Reference Intervals for Both Methods}

The reference intervals were defined as the 2.5 and 97.5 percentiles $[20,21]$. The HPLC reference intervals were calculated for the flavins. FAD values below $26.2 \mathrm{nM}$, FMN below $6.7 \mathrm{nM}$, or riboflavin level below $2.2 \mathrm{nM}$ were deemed riboflavin deficient. The EGRAC reference interval was calculated to be from $0.93-1.59$, thus any sample with an activity coefficient $>1.59$ was deemed riboflavin deficient (Table 1). Previous reports suggest an activity coefficient threshold for riboflavin deficiency of between $>1.2$ and $>1.7$ [23].

\section{Method Comparison}

FAD levels assessed by HPLC and EGRAC correlated well $(r=-0.935, p<0.001)$ (See Figs. 3a-3c). However, there was a poorer correlation between HPLC and EGRAC measurements of riboflavin $(r=-0.382, p<0.001)$, and FMN $(r=-$ $0.706, p<0.001)$, although these were still highly significant. After evaluating both methods, the HPLC method was selected as the method of choice, for assessing riboflavin levels in TPN patients, principally because of its greater sensitivity (LOD), larger number of analytes detected, and better reproducibility.

\section{Reference Population Comparison with TPN Patients}

There was no significant difference between FAD and FMN levels in the TPN and control populations (Table 2). However, there was a statistically significant difference between the riboflavin levels in the patient and control groups $(\mathrm{p}<0.001)$, with the TPN patients having higher levels of riboflavin than the reference population. 
Table 2. Comparison between Flavin Levels in the Reference Population and TPN Patients. Data are Expressed as Median and Interquartile Ranges. (n.s = non significant)

\begin{tabular}{|c|c|c|c|}
\hline & Control Population (n=90) & TPN Patients (n=51) & Significance \\
\hline \hline FAD (nM) & $51.4(39.2-62.9)$ & $45.5(34.0-56.7)$ & n.s. \\
\hline FMN (nM) & $15.5(11.6-20.9)$ & $16.2(12.0-19.9)$ & n.s. \\
\hline Riboflavin (nM) & $7.6(5.5-12.1)$ & $20.4(11.7-31.5)$ & $\mathrm{p}<0.001$ \\
\hline
\end{tabular}

There are few possible riboflavin analogues that could be used as internal standard in this method, such as galactoflavin and isoriboflavin. However, as we were unable to obtain either of these products we used lumiflavin, a known riboflavin analogue, which was readily available. Several papers have discussed the possibility that lumiflavin is a photodegradation product of riboflavin [16,19], so we assessed this using our method. Aliquots of the same plasma sample were spiked with FAD, FMN, riboflavin, and lumiflavin and exposed to full range UV light for varying lengths of time (1560 minutes). The flavins were then extracted and analysed by HPLC (See Fig. 2).

It is clear that FMN (peak 2), and riboflavin (peak 3) are degraded rapidly as the peaks decrease in size quite dramatically after 30 minutes of exposure to UV light. FAD (peak 1) appears to be more resistant to degradation by UV light as there is not as large a decrease in peak size compared to riboflavin or FMN. Levels of lumiflavin (peak 4) do not increase suggesting that riboflavin does not degrade to lumiflavin under these conditions. There is also an extra peak (peak X) which appears next to lumiflavin on the chromatogram after exposure of the sample to UV light. This unidentified peak appears to be a degradation product with a similar, but chromatographically distinct, profile to lumiflavin, and it is possible that this may have been mistaken for lumiflavin in previous studies.

As the preparation of samples from venesection to injection onto the HPLC column was performed in dim light, it was decided that lumiflavin was suitable to use as internal standard for this method.

\section{DISCUSSION}

There was a strongly significant correlation between the EGRAC and HPLC methods with regard to FAD levels, with a significant correlation also found for FMN and riboflavin. These data are in some agreement with results published by Moat et al. [24] who found a strong correlation between plasma riboflavin assessed by HPLC and the EGRAC assay, although they reported no significant correlation between FMN and FAD assessed by HPLC and EGRAC. We found a weaker, but still statistically significant association between

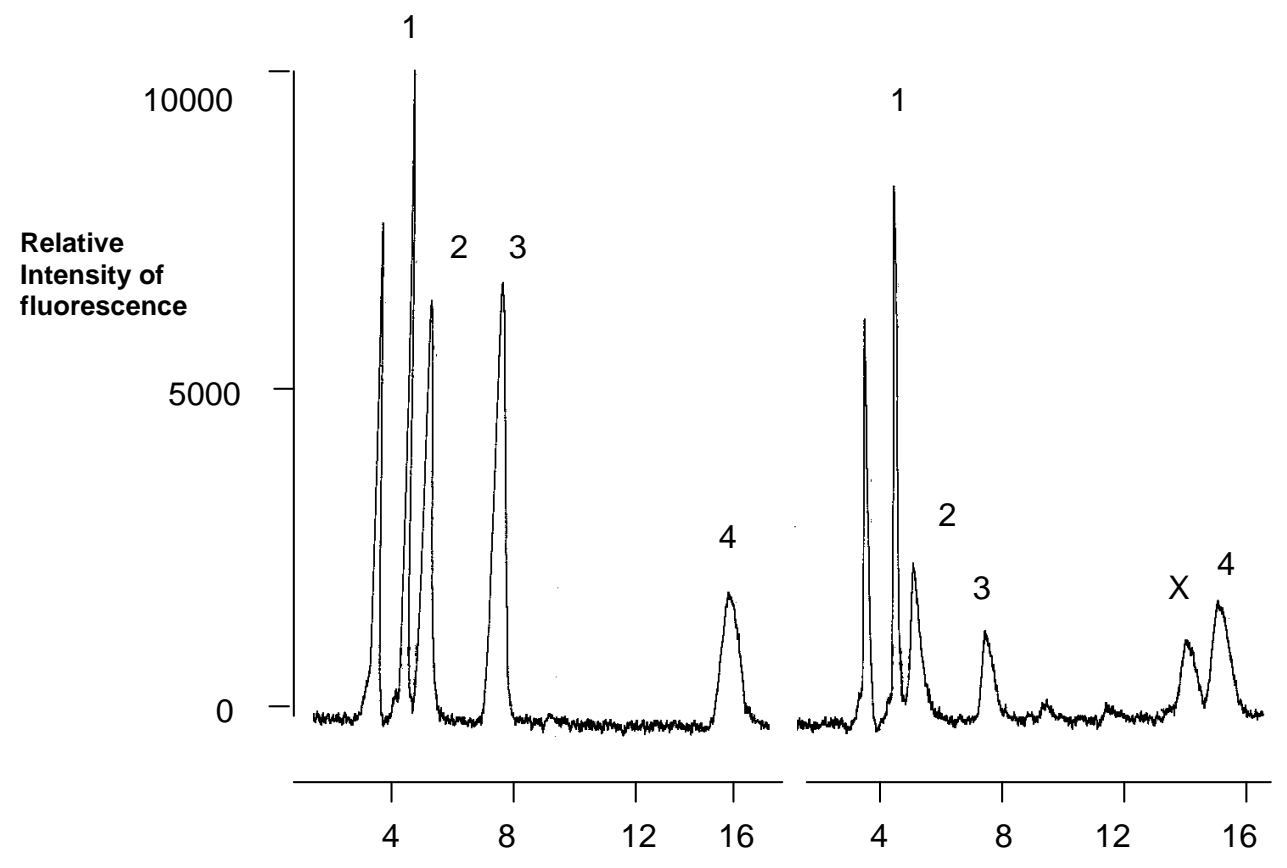

Time (minutes)

Fig. (2). Chromatograms of the flavins in spiked plasma before and after exposure to UV light for 30 minutes.

a. Before exposure to UV light.

b. After exposure to UV light. 
a

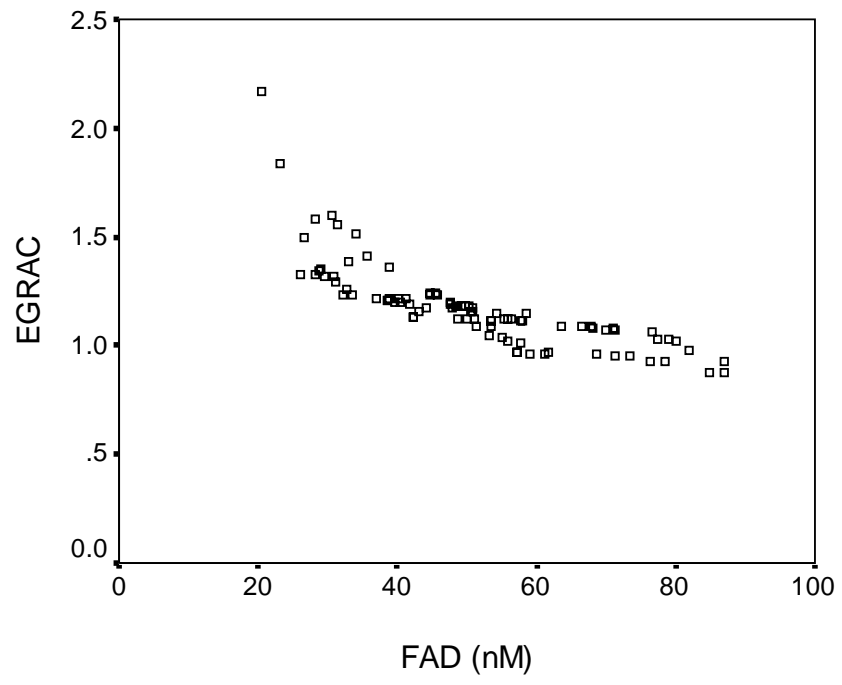

b

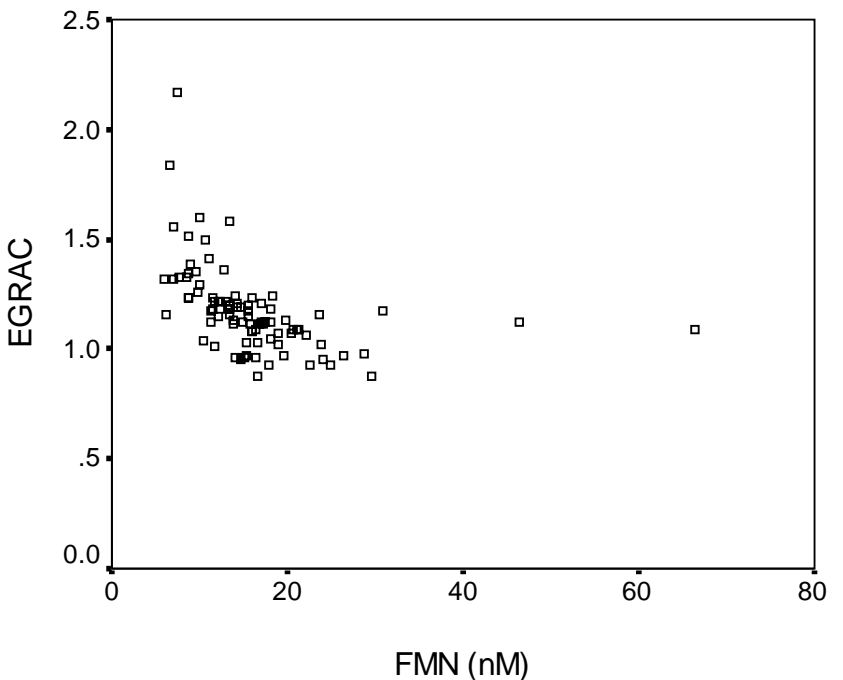

c

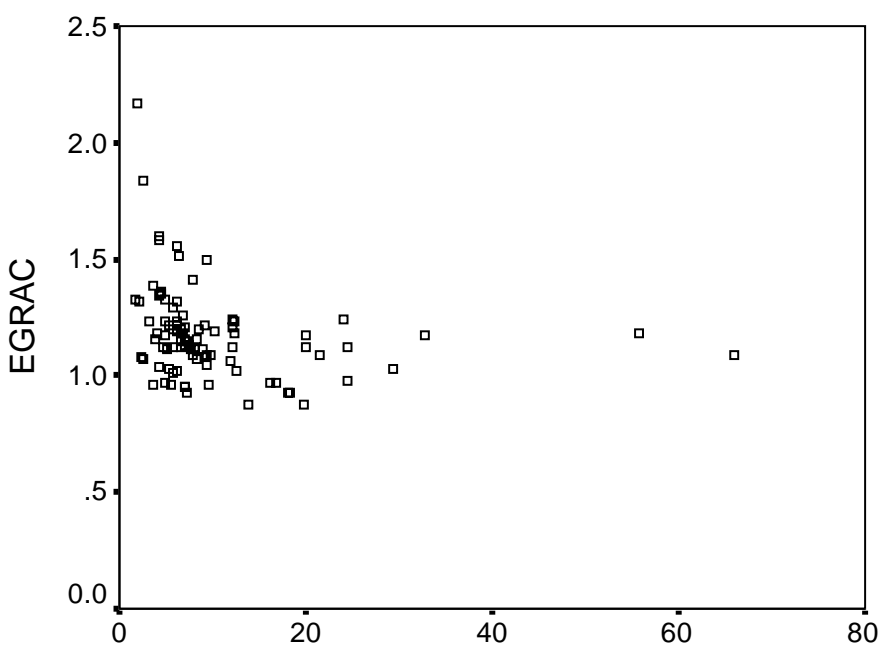

Riboflavin (nM)

Fig. (3). a. Correlation between EGRAC and FAD ( $p<0.001)$.

b. Correlation between EGRAC and FMN ( $p<0.001)$

c. Correlation between EGRAC and riboflavin $(\mathrm{p}<0.001)$.

FMN and FAD assessed by HPLC and EGRAC, and the reason for this contrast with the Moat study [24] is unclear. The results of the present study suggest that either method would be suitable for routine assessment of flavin status in the biochemistry laboratory. However, the HPLC method, which measures FAD, FMN and riboflavin simultaneously, is more informative in that it gives a complete overall assessment of flavin status compared to the EGRAC method (Table 1). The HPLC method is more sensitive with a lower limit of detection than the EGRAC method, which is potentially an important factor when assessing riboflavin deficiency. In addition, the HPLC method is not subject to interference in the same way as the EGRAC method. Glucose-6phosphate dehydrogenase deficiency and diabetes mellitus may both produce misleading results in the EGRAC assay, whereas the HPLC method is unaffected by such disorders $[3,4,18]$.
There has been considerable controversy as to what the results of the EGRAC assay actually show, and whether they reflect short term or long term dietary intake. The EGRAC method utilises the role of riboflavin as a cofactor in glutathione reductase synthesis to estimate the amount of riboflavin present in a sample, and is considered by some to represent a more accurate picture of long-term riboflavin status than direct measurement [17]. It has been stated that while the EGRAC activity responds quickly to acute riboflavin deficiency, there are doubts as to whether it continues to change in response to progressive tissue depletion [9]. By contrast, several reports claim that the EGRAC reflects accurately the long-term intake of riboflavin but is of limited use in assessing the kinetics of riboflavin in the short-term [25, 26].

As FAD is conserved at the expense of riboflavin [27] a decrease in the levels of FMN or riboflavin may be evident 
before levels of FAD would begin to decrease in the early stages of riboflavin deficiency. This means that the HPLC method is more useful than the EGRAC assay, which only measures FAD levels, in the detection of mild riboflavin deficiency.

The levels of FAD required to drive the EGRAC reaction may not be the same as the concentrations needed for other physiological biochemical reactions, meaning that an individual suffering from mild riboflavin deficiency may not necessarily have an abnormal EGRAC. HPLC could also be more sensitive to changes in riboflavin status in that large changes at certain points of the EGRAC standard curve may cause only small changes in the EGRAC value, whereas the HPLC standard curves are linear and may therefore reflect small changes better than the EGRAC.

The HPLC method described in this study would be able to detect excess amounts of riboflavin, whereas the EGRAC assay is relatively insensitive to high levels. Many vitamins can be damaging to the body at high levels. Vitamins A, C, and $\mathrm{E}$ are all known to potentially generate reactive oxygen species when consumed in high doses [28-30]. To date, there have been no reports of any significant adverse structural or functional effects of excess riboflavin consumption in humans. The lack of any harmful effects from high doses of riboflavin may be due to its physicochemical properties. Its solubility is limited and the capacity to absorb riboflavin via the gastrointestinal tract is limited, particularly when administered in large doses [31]. Once the tissues are saturated, any excess riboflavin is excreted in the urine.

However, this does not mean that there is no potential for adverse effects from high intakes [32]. Riboflavin has been implicated in toxicity when taken as part of a standard multivitamin preparation. In this situation, riboflavin may be involved in photodynamic damage to DNA, but only in synergism with other multivitamin components, because it has been shown that high levels of riboflavin alone are unable to damage DNA [33].

With regard to the costs and ease of use of the methods, both require expensive equipment in the first instance, with the EGRAC assay being performed on a centrifugal analyser such as the Cobas Fara and the HPLC method requiring an HPLC pump, fluorescence detector, and data system. Both methods require a similar amount of preparation time, although an automatic sampler would be required in order to facilitate the analysis of a similar number of samples through the HPLC assay compared to the EGRAC method on a daily basis. The costs of reagents needed for each method are also similar.

There were no significant differences between the FAD and FMN levels in patients receiving TPN and control subjects, suggesting that riboflavin deficiency is not prevalent in this patient group. However, in the case of riboflavin, there was a significant difference between the TPN patients and the controls, indicating that the TPN patients were receiving more riboflavin than necessary. In such patients, an increased availability of riboflavin could be beneficial in that they may have an increased requirement for FAD to aid in the recovery process [13].

In conclusion, the HPLC method evaluated in this study is superior to the EGRAC assay for assessing riboflavin status. Riboflavin deficiency is not prevalent in TPN patients.

\begin{tabular}{|c|c|c|}
\hline \multicolumn{3}{|c|}{ ABBREVIATIONS } \\
\hline ATP & $=$ & Adenosine triphosphate \\
\hline $\mathrm{CV}$ & $=$ & Coefficient of variation \\
\hline DNA & $=$ & Deoxyribonucleic acid \\
\hline EDTA & $=$ & Ethylene diamine tetraacetic acid \\
\hline EGRAC & $=$ & $\begin{array}{l}\text { Erythrocyte glutathione reductase activity co- } \\
\text { efficient }\end{array}$ \\
\hline FAD & $=$ & Flavin adenine dinucleotide \\
\hline FMN & $=$ & Flavin mononucleotide \\
\hline EGR & $=$ & Glutathione reductase \\
\hline GSH & $=$ & Reduced glutathione \\
\hline GSSG & $=$ & Oxidised glutathione \\
\hline HPLC & $=$ & High performance liquid chromatography \\
\hline LOD & & Limit of detection \\
\hline $\mathrm{NADP}^{+}$ & $=$ & $\begin{array}{l}\text { Nicotinamide adenine dinucleotide phosphate } \\
\text { (oxidised) }\end{array}$ \\
\hline NADPH & $=$ & $\begin{array}{l}\text { Nicotinamide adenine dinucleotide phosphate } \\
\text { (reduced) }\end{array}$ \\
\hline TPN & $=$ & Total parenteral nutition \\
\hline UV & $=$ & Ultraviolet \\
\hline
\end{tabular}

\section{AUTHORS' CONTRIBUTIONS}

JSH performed the laboratory analyses and drafted the manuscript. JVW participated in the design of the study, performed the statistical analyses, and helped draft the final manuscript. WAMcI set up the EGRAC method for use in the study. MCM was involved in the design and interpretation of the study and helped draft the final manuscript. ISY conceived the study and participated in its design and coordination and helped draft the final manuscript. All authors read and approved the final manuscript.

\section{ACKNOWLEDGEMENTS}

This project was funded by a Research Scholarship from the Association for Clinical Biochemistry Scientific Committee. This study was presented as a thesis for the award of MSc. Many thanks to the patients and volunteers who provided blood samples for this study.

\section{REFERENCES}

[1] Powers, H.J. Riboflavin (vitamin-B2) and health. Am. J. Clin. Nutr., 2003, 77, 1352.

[2] Capo-chichi, C.D.; Feillet, F.; Gueant, J.-L.; Amouzou, K.S.; Zonon, N.; Sanni, A.; Lefebvre, E.; Assimadi, K.; Vidailhet, M. Concentrations of riboflavin and related organic acids in children with protein-energy malnutrition. Am. J. Clin. Nutr., 2000, 71, 978-986.

[3] Basu, T.K.; Dickerson, J.W. Vitamins in Human Health and Disease. $\mathrm{CAB}$ International: Oxon, $\mathrm{UK}, 1996$.

[4] Capo-chichi, C.D.; Gueant, J.-L.; Feillet, F.; Namour, F.; Vidailhet, M. Analysis of riboflavin and riboflavin cofactor levels in plasma by high-performance liquid chromatography. J. Chromatogr. B, 2000, 739, 219.

[5] McCormick, D.B. Two interconnected B vitamins: Riboflavin and pyridoxine. Physiol. Rev., 1989, 69, 1107. 
[6] Grimble, R.F. Modification of inflammatory aspects of immune function by nutrients. Nutr. Res., 1998, 18, 1297.

[7] Rozen, R. Methylenetetrahydrofolate reductase: a link between folate and riboflavin? Am. J. Clin. Nutr., 2002, 76, 301.

[8] McNulty, H.; McKinley, M.C.; Wilson, B.; McPartlin, J.; Strain, JJ.; Weir, DG.; Scott, J.M. Impaired functioning of thermolabile methyltetrahydrofolate reductase is dependent on riboflavin status: implications for riboflavin requirements. Am. J. Clin. Nutr., 2002, 76,436 .

[9] Nichoalds, G.E. Riboflavin. Clin. Lab. Med., 1981, 1, 685.

[10] Mikalunas, V.; Fitzgerald, K.; Rubin, H.; McCarthy, R.; Craig, R.M. Abnormal vitamin levels in patients receiving home total parenteral nutrition. J. Clin. Gastroenterol., 2001, 33, 393.

[11] Friel, J.K.; Bessie, J.C.; Belkhode, S.L.; Edgecombe, C.; SteeleRodway, M.; Downton, G.; Kwa, PG.; Aziz, K. Thiamine, riboflavin, pyridoxine, and vitamin $\mathrm{C}$ status in premature infants receiving parenteral and enteral nutrition. J. Pediatr. Gastroenterol. Nutr., 2001, 33, 64 .

[12] Burtis, C.A.; Ashwood, E.R. Tietz Textbook of Clinical Chemistry, 2nd ed.; W.B. Saunders Co.; Philadelphia, 1994.

[13] Huang, S.I.; Caldwell, M.J.; Simpson, K.L. Urinary riboflavin determination by $\mathrm{C} 18$ reversed-phase open-column chromatography. Methods Enzymol., 1997, 280, 343.

[14] Lambert, W.E.; Cammaert, P.M.; De Leenheer, A.P. Liquidchromatographic measurement of riboflavin in serum and urine with isoriboflavin as internal standard. Clin. Chem., 1985, 31, 1371.

[15] Russell, L.F.; Vanderslice, J.T. A comprehensive review of vitamin B2 analytical methodology. J. Micronutr. Anal., 1990, 8, 257.

[16] Russell, L.F.; Brooks, L.; McRae, K.B. Development of a roboticHPLC determination of riboflavin vitamers in food. Food Chem., 1998, 63, 125 .

[17] Sauberlich, H.E.; Judd, J.H.; Nichoalds, G.E.; Broquist, H.P.; Darby, W.J. Application of the erythrocyte glutathione reductase assay in evaluating riboflavin nutritional status in a high school student population. Am. J. Clin. Nutr., 1972, 25, 756.

[18] Hamilton, J.S.; Powell, L.A.; McMaster, C.; McMaster, D.; Trimble, E.R. Interaction of glucose and long chain fatty acids (C18) on antioxidant defences and free radical damage in porcine vascular smooth muscle cells in vitro. Diabetologia, 2003, 46, 106.

[19] Hustad, S.; Ueland, P.M.; Schneede, J. Quantification of riboflavin, FMN, and FAD in human plasma by capillary electrophoresis and laser-induced fluorescence detection. Clin. Chem., 1999, 45, 862.

[20] Jones, R.G.; Payne, R.B. Clinical Investigation and Statistics in Laboratory Medicine. ACB Venture Publications: London, UK, 1997.
[21] International Federation of Clinical Chemistry (IFCC). Approved Recommendation on the theory of reference values. Part 5. Statistical treatment of collected reference values. Determination of reference limits. J. Clin. Chem. Clin. Biochem., 1987, 25, 645.

[22] Powers, H.J. Investigation into the relative effects of riboflavin deprivation on iron economy in the weanling rat and the adult. Ann Nutr. Metab., 1986, 30, 308

[23] Dror, Y.; Stern, F.; Komarnitsky, M. Optimal and stable conditions for the determination of erythrocyte glutathione reductase activation coefficient to evaluate riboflavin status. Int. J. Vitam. Nutr. Res., 1996, 64, 257.

[24] Moat, S.J.; Ashfield-Watt, P.A.L.; Powers, H.J.; Newcombe, R.G.; McDowell, I.F.W. Effect of riboflavin status on the homocysteinelowering effect of folate in relation to the MTHFR (C677T) genotype. Clin. Chem. 2003, 49, 295-302.

[25] Zempleni, J. Determination of riboflavin and flavocoenzymes in human blood plasma by high-performance liquid chromatography. Ann. Nutr. Metab., 1995, 39, 224.

[26] Bates, C. Riboflavin. Int. J. Vitam. Nutr. Res., 1993, 63, 274.

[27] Floridi, A.; Palmerini, C.A.; Fini, C.; Pupita, M.; Fidanza, F. High performance liquid chromatographic analysis of flavin adenine dinucleotide in whole blood. Int. J. Vitam. Nutr. Res., 1985, 55 , 187.

[28] Burton, G.W.; Ingold, K.U. $\beta$-carotene: an unusual type of lipid antioxidant. Science, 1984, 224, 569.

[29] Stahl, W.; Sies, H. Antioxidant defence: Vitamins E and C and carotenoids. Diabetes, 1997, 46, S14.

[30] Van den Berg, J.J.M.; Kuypers, F.A.; Roelofsen, B.; Op den Kamp, J.A.F. The cooperative action of vitamins $\mathrm{E}$ and $\mathrm{C}$ in the protection against peroxidation of parinaric acid in human erythrocyte membranes. Chem. Physiol. Lipids, 1990, 53, 309.

[31] Zempleni, J.; Galloway, J.R.; McCormick, D.B. Pharmakokinetics of orally and intravenously administered riboflavin in healthy humans. Am. J. Clin. Nutr., 1996, 63, 54.

[32] European Commission, 2000. Health \& Consumer Protection Directorate-General. Opinion of the Scientific Committee on Food on the tolerable upper intake level of VitaminB2. Available from. http://europa.eu.int/comm/food/fs/sc/scf/index_en.html [accessed July 21, 2008]

[33] Ennever, J.F.; Carr, H.S.; Speck, W.T. Potential for genetic damage from multivitamin solutions exposed to phototherapy illumination. Paediatr. Res., 1983, 17, 192.

(C) Hamilton et al.; Licensee Bentham Open.

This is an open access article licensed under the terms of the Creative Commons Attribution Non-Commercial License (http://creativecommons.org/licenses/by-nc/3.0/) which permits unrestricted, non-commercial use, distribution and reproduction in any medium, provided the work is properly cited. 